\title{
Virtual screening and optimization of Type II inhibitors of JAK2 from a natural product library†
}

\author{
Dik-Lung Ma ${ }^{a}$, Daniel Shiu-Hin Chan ${ }^{a}$, Guo Wei ${ }^{b}$, Hai-Jing Zhong ${ }^{c}$, Hui Yang ${ }^{a}$, Lai To \\ Leung $^{b}$, Elizabeth A. Gullen ${ }^{d}$, Pauline Chiu ${ }^{b}$, Yung-Chi Cheng ${ }^{d}$, and Chung-Hang Leung ${ }^{c}$ \\ Dik-Lung Ma: edmondma@hkbu.edu.hk; Pauline Chiu: pchiu@hku.hk; Yung-Chi Cheng: yccheng@yale.edu; Chung- \\ Hang Leung: duncanleung@umac.mo \\ aDepartment of Chemistry, Hong Kong Baptist University, Kowloon Tong, Hong Kong, China \\ ${ }^{b}$ Department of Chemistry and the State Key Laboratory of Synthetic Chemistry, The University of \\ Hong Kong, Pokfulam Road, Hong Kong, China \\ 'State Key Laboratory of Quality Research in Chinese Medicine, Institute of Chinese Medical \\ Sciences, University of Macau, Macao, China \\ dDepartment of Pharmacology, Yale University School of Medicine, New Haven, CT 06520, USA
}

\section{Abstract}

\begin{abstract}
Amentoflavone has been identified as a JAK2 inhibitor by structure-based virtual screening of a natural product library. In silico optimization using the DOLPHIN model yielded analogues with enhanced potency against JAK2 activity and HCV activity in cellulo. Molecular modeling and kinetic experiments suggested that the analogues may function as Type II inhibitors of JAK2.
\end{abstract}

Hepatitis $\mathrm{C}$ is a highly infectious disease affecting the liver, caused by the hepatitis $\mathrm{C}$ virus (HCV). ${ }^{1}$ Chronic HCV infection could lead to liver fibrosis and cirrhosis, which could eventually result in liver failure and/or other complications, including liver cancer. HCV non-structural proteins induce activation of signal transducer and activator of transcription 3 (STAT3), leading to constitutive activation of STAT3 in HCV replicon-expressing cells. ${ }^{2-4}$ Interestingly, treatment of HCV-infected cells with Janus kinase 2 (JAK2) ${ }^{5}$ inhibitor AG490 decreased STAT3 activity and consequently, HCV RNA production. ${ }^{2}$ This suggests that novel JAK2 inhibitors could also inhibit HCV translation and replication, potentially supplementing existing treatment for HCV.

The vast majority of protein kinase inhibitors discovered so far are Type I inhibitors, as they primarily bind in and around the ATP-binding site of the kinases in their active "DFG-in" conformation, where the highly conserved Asp-Phe-Gly (DFG) motif of the activation loop is oriented towards the binding site. ${ }^{6}$ In contrast, Type II inhibitors such as imatinib (Gleevec), ${ }^{7}$ BIRB $796^{8}$ and sorafenib ${ }^{9}$ also target a hydrophobic pocket vacated by the movement of the phenylalanine residue of the DFG motif away from its position in the

\footnotetext{
$\dagger$ Electronic Supplementary Information (ESI) available: compound characterisation and supplementary data. See DOI: 10.1039/ c000000x/

Correspondence to: Dik-Lung Ma, edmondma@hkbu.edu.hk; Pauline Chiu, pchiu@hku.hk; Yung-Chi Cheng, yccheng@yale.edu; Chung-Hang Leung, duncanleung@umac.mo.
} 
active conformation. It has been proposed that Type II inhibitors may achieve greater selectivity for target kinases due to the greater structural heterogeneity of the hydrophobic pocket in the DFG-out conformation compared to the ATP-binding site. ${ }^{6}$ Radimerski and co-workers have recently shown that NVP-BBT594, a potent Type II inhibitor of wild-type and T315I mutant Bcr-Abl, also binds to JAK2 in the DFG-out conformation. ${ }^{10}$ To our knowledge, no other Type II inhibitors of JAK2 have been reported in the literature. In this study, we proposed to utilize a structure-based lead optimization approach to generate novel natural product-like Type II inhibitors of JAK2 using the DOLPHIN protocol. We initially docked a panel of known JAK2 inhibitors against twelve X-ray crystal structures of JAK2. The X-ray co-crystal structure of JAK2 with the pan-Janus kinase inhibitor CMP6 (PDB code: $2 \mathrm{~B} 7 \mathrm{~A})^{11}$ was deemed to be the most predictive structure according to our molecular modeling methods as it yielded the highest average docking score. However, no X-ray crystal structure of JAK2 in the inactive conformation was available at the onset of this study. Therefore, we used the DOLPHIN protocol developed by Abagyan and co-workers ${ }^{12}$ to convert the aforementioned structure into an inactive conformation suitable for the molecular docking-based screening of Type II JAK2 inhibitors. After the generation of the DOLPIN kinase model, we performed in silico screening of natural product and natural product-like databases using the ICM method. The top eleven highest-scoring compounds were genterated from the initial high-throughput virtual screening campaign (Fig. S1). Amentoflavone 1a (Fig. 1), a biflavonoid from the Chinese plant Gingko biloba, emerged as a promising candidate for further biological evaluation. In dose-response experiments, amentoflavone 1a inhibited JAK2 activity in a concentration-dependent fashion with an $\mathrm{IC}_{50}$ value of $5 \mu \mathrm{M}$ (Fig. S2).

We next utilized a computer-aided lead optimization strategy to develop more potent JAK2 inhibitors using 1a as a lead structure. We observed that the deletion of the DFG loop in the DOLPHIN model of JAK2 kinase revealed a large hydrophobic pocket, not present in the active DFG-in form of the kinase, that was partially occupied by amentoflavone 1a (Fig. 2a). We envisaged that the addition of one or more aliphatic side chains to the biflavonoid scaffold of 1 a could more fully occupy the hydrophobic pocket vacated by the DFG loop. As a proof-of-concept, we designed nine such analogues (1b-j) (Fig. 1) and docked these against the DFG-out model of JAK2 kinase in silico. Our molecular modeling analysis indicated that the long aliphatic side chain of the highest-scoring analogue 1c extended deep into the hydrophobic pocket, which probably contributed to the high binding energy $(-35.73$ $\mathrm{kcal} / \mathrm{mol}$ ) of this compound (Fig. 2b). The structures and binding scores of analogues $\mathbf{1 b}-\mathbf{j}$ are depicted in Table S1.

However, the hexyl $\left(\mathrm{C}_{6}\right)$ analogue 1c was found to be relatively toxic towards cells (see below), which was deemed to be undesirable for the development of anti-HCV therapeutics. Hence, we also studied the interaction of the octyl $\left(\mathrm{C}_{8}\right)$ analogue $\mathbf{1 b}(-31.59 \mathrm{kcal} / \mathrm{mol})$ with the inactive conformation of JAK2 by molecular modeling (Fig. 2c). Similar to analogue 1c, the amentoflavone moiety of $\mathbf{1 b}$ was located in the ATP binding pocket, whereas the alkyl side chain protruded inward into the hydrophobic pocket adjacent to the ATP binding site. For comparison, the interaction between $\mathbf{1 b}$ and $\mathbf{1 c}$ with the active conformation of JAK2 was also studied. The less favorable ICM binding scores $(c a .-10 \mathrm{kcal} / \mathrm{mol})$ for those 
complexes suggested that the binding between $\mathbf{1 b}$ and $\mathbf{1 c}$ to the active form of JAK2 is relatively weak.

The procedures to synthesise the novel amentoflavone analogues $\mathbf{1 b}-\mathbf{j}$ and their characterization are detailed in the ESI. (Scheme S1). The cytotoxicity of the amentoflavone analogues against HEL cells was determined by the MTT assay. The results revealed that the hexyl $\left(\mathrm{C}_{6}\right)$ analogue $1 \mathrm{c}$ showed relatively pronounced effects on cell viability compared to the other tested compounds, with an $\mathrm{IC}_{50}$ value of $0.62 \mu \mathrm{M}$ (Fig. S3 and Table S2). On the other hand, the octyl $\left(\mathrm{C}_{8}\right)$ analogue $\mathbf{1 b}$ was found to be relatively non-toxic towards HEL cells $\left(\mathrm{IC}_{50}>100 \mu \mathrm{M}\right)$.

The activation of STAT3 by HCV non-structural proteins is required for HCV viral replication, and inhibitors of JAK2 have been reported to suppress HCV RNA production. ${ }^{2}$ Therefore, the antiviral activity of the control compound NVP-BBT594 and compounds 1ac was tested in the HCV replicon (Huh-Luc/neo-ET) cell line. The results showed that the octyl $\left(\mathrm{C}_{8}\right)$ analogue $\mathbf{1 b}$ was highly potent against $\mathrm{HCV}$ activity in vitro, with an $\mathrm{EC}_{50}$ value of $3.1 \pm 0.8 \mu \mathrm{M}$ (Table 1). Significantly, compound $\mathbf{1 b}$ was more potent at suppressing HCV activity compared to the parent compound, amentoflavone $1 \mathbf{a}\left(\mathrm{EC}_{50}>50 \mu \mathrm{M}\right)$. The Type II JAK2 inhibitor NVP-BBT594 also displayed potent inhibition of HCV activity $\left(\mathrm{EC}_{50}<1.5\right.$ $\mu \mathrm{M})$.

Given the promising antiviral activity exhibited by the octyl $\left(\mathrm{C}_{8}\right)$ analogue $\mathbf{1 b}$, its potency against JAK2 activity was further investigated by a dose-response experiment. The results revealed that analogue $\mathbf{1 b}$ inhibited JAK2 activity in a concentration-dependent fashion, with an $\mathrm{IC}_{50}$ value of $3.9 \mu \mathrm{M}$ (Fig. S4). Notably, the potency of $\mathbf{1 b}$ was superior to that of the parent compound, amentoflavone $\mathbf{1 a}$.

The effect of compound $\mathbf{1 b}$ on JAK2 autophosphorylation in cellulo was further tested using a Western blot assay in human erythroleukemia cells (HEL). Compound $\mathbf{1 b}$ exhibited a dose-dependent reduction of JAK2 autophosphorylation, with comparable potency to the control compound JAK2 Inhibitor II (Fig. 3). We postulate that the HCV antiviral activity of compound $\mathbf{1 b}$ could be attributed, at least in part, to the inhibition of JAK2 signaling in cells, thereby leading to reduced STAT3 activity and HCV.

To develop a preliminary structure-activity relationship (SAR) hypothesis, the compounds $\mathbf{1 a}-\mathbf{j}$ were tested for JAK2 inhibitory activity in the same manner. The results revealed that unsubstituted compounds or those with medium-length $\left(\mathrm{C}_{6}-\mathrm{C}_{8}\right)$ alkyl side chains $(\mathbf{1 a}, \mathbf{1 b}$ and 1c) showed better inhibition than those with longer alkyl side chains $\left(\geq_{10}\right)$ (Fig. S5). We envisage that amentoflavone analogues with excessively long side chains were unable to fit effectively within the JAK2 ATP-binding pocket, leading to reduced inhibition of JAK2 autophosphorylation activity.

Given the ability of the octyl $\left(\mathrm{C}_{8}\right)$ analogue $\mathbf{1 b}$ to inhibit JAK2 phosphorylation in HEL cells, we investigated whether compound $\mathbf{1 b}$ could suppress STAT3 activation in cellulo. The results revealed a dose-dependent decrease in STAT3 phosphorylation in treated HEL cells as the concentration of $\mathbf{1 b}$ was increased, while no significant effect was observed on 
total STAT3 expression (Fig. 4). The inhibition of STAT3 phosphorylation by compound $\mathbf{1 b}$ was observable at $2.5 \mu \mathrm{M}$, and its potency was comparable to that of the positive control compound S3I-201, a small molecule inhibitor of STAT3 dimerization, under the same conditions. We envision that the inhibition of STAT3 activation by compound $\mathbf{1 b}$ in cellulo could be attributed, at least in part, to the inhibition of JAK2 activity by compound $\mathbf{1 b}$. The reduction of STAT3 activation could potentially repress signaling pathways required for viral replication, thus leading to the observed inhibition of $\mathrm{HCV}$ in cellulo.

Kinetic studies were performed on the octyl $\left(\mathrm{C}_{8}\right)$ analogue $\mathbf{1 b}$ using ELISA to elucidate the mechanism of JAK2 inhibition by the amentoflavone analogues. Lineweaver-Burke analysis suggested that compound $\mathbf{1 b}$ functioned as a non-ATP-competitive inhibitor of JAK2 (Fig. $5)$. Plotting the vertical intercept $\left(1 / V_{\mathrm{m}}\right)$ of each line against the inhibitor concentration (Dixon plot) yields an estimated $K_{\mathrm{i}}$ value of $27 \mu \mathrm{M}$ (Fig. 5 inset). The Type II JAK2 inhibitor NVP-BBT594 ${ }^{10}$ also displayed non-ATP-competitive behaviour under the same conditions (Fig. S6), with an estimated $K_{\mathrm{i}}$ value of $22 \mu \mathrm{M}$. Given that Type II inhibitors are known to be non-competitive with ATP, ${ }^{13}$ these data suggest that compound $\mathbf{1 b}$ may be putatively considered as a Type II inhibitor of JAK2.

In conclusion, the DOLHPIN computational protocol was employed to convert an active (DFG-in) conformation of JAK2 into a Type II-compatible conformation, which was used as a model for the structure-based virtual screening of 150,000 natural product-like compounds in silico. By conducting in silico lead optimisation on the hit compound amentoflavone 1a, the novel biflavonoid derivatives $\mathbf{1 b}-\mathbf{j}$ were synthesised and then tested for JAK2 and STAT3 inhibitory activity, cytotoxicity and HCV antiviral activity. The octyl $\left(\mathrm{C}_{8}\right)$ analogue 1b displayed superior potency against JAK2 activity and $\mathrm{HCV}$ activity compared to the parent compound 1a, validating the structure-based lead optimisation approach used in this study. Moreover, kinetic analysis indicated that analogue $\mathbf{1 b}$ exhibited a non-competitive mode of inhibition, suggesting that this compound may be a putative Type II inhibitor of JAK2.

\section{Supplementary Material}

Refer to Web version on PubMed Central for supplementary material.

\section{Acknowledgments}

This work is supported by Hong Kong Baptist University (FRG2/12-13/021 and FRG2/13-14/008), Centre for Cancer and Inflammation Research, School of Chinese Medicine (CCIR-SCM, HKBU), the Health and Medical Research Fund (HMRF/11101212 and HMRF/13121482), the Research Grants Council (HKBU/201811, HKBU/ 204612 and HKBU/201913), the French National Research Agency/Research Grants Council Joint Research Scheme (A-HKBU201/12), the State Key Laboratory of Synthetic Chemistry, the Science and Technology Development Fund, Macao SAR (103/2012/A3) and the University of Macau (MYRG091(Y3-L2)-ICMS12-LCH, MYRG121(Y3-L2)-ICMS12-LCH, MRG007/LCH/2014/ICMS and MRG023/LCH/2013/ICMS). We especially thank Novartis Pharma AG for providing NVP-BBT594 for the biological assays.

\section{References}

1. Tian Z. Nat Immunol. 2014; 15:6-8. [PubMed: 24352316]

2. Waris G, Turkson J, Hassanein T, Siddiqui A. J Virol. 2005; 79:1569-1580. [PubMed: 15650183] 
3. Hosui A, Ohkawa K, Ishida H, Sato A, Nakanishi F, Ueda K, Takehara T, Kasahara A, Sasaki Y, Hori M, Hayashi N. J Biol Chem. 2003; 278:28562-28571. [PubMed: 12764155]

4. Yoshida T, Hanada T, Tokuhisa T, Kosai K, Sata M, Kohara M, Yoshimura A. J Exp Med. 2002; 196:641-653. [PubMed: 12208879]

5. Ghoreschi K, Laurence A, O'Shea JJ. Immunol Rev. 2009; 228:273-287. [PubMed: 19290934]

6. Liu Y, Gray NS. Nat Chem Biol. 2006; 2:358-364. [PubMed: 16783341]

7. Schindler T, Bornmann W, Pellicena P, Miller WT, Clarkson B, Kuriyan J. Science. 2000; 289:1938-1942. [PubMed: 10988075]

8. Pargellis C, Tong L, Churchill L, Cirillo PF, Gilmore T, Graham AG, Grob PM, Hickey ER, Moss N, Pav S, Regan J. Nat Struct Biol. 2002; 9:268-272. [PubMed: 11896401]

9. Wan PT, Garnett MJ, Roe SM, Lee S, Niculescu-Duvaz D, Good VM, Jones CM, Marshall CJ, Springer CJ, Barford D, Marais R. Cell. 2004; 116:855-867. [PubMed: 15035987]

10. Andraos R, Qian Z, Bonenfant D, Rubert J, Vangrevelinghe E, Scheufler C, Marque F, Regnier CH, De Pover A, Ryckelynck H, Bhagwat N, Koppikar P, Goel A, Wyder L, Tavares G, Baffert F, Pissot-Soldermann C, Manley PW, Gaul C, Voshol H, Levine RL, Sellers WR, Hofmann F, Radimerski T. Cancer Discov. 2012; 2:512-523. [PubMed: 22684457]

11. Lucet IS, Fantino E, Styles M, Bamert R, Patel O, Broughton SE, Walter M, Burns CJ, Treutlein H, Wilks AF, Rossjohn J. Blood. 2006; 107:176-183. [PubMed: 16174768]

12. Kufareva I, Abagyan R. J Med Chem. 2008; 51:7921-7932. [PubMed: 19053777]

13. Blanc J, Geney R, Menet C. Anticancer Agents Med Chem. 2013; 13:731-747. [PubMed: 23094911] 


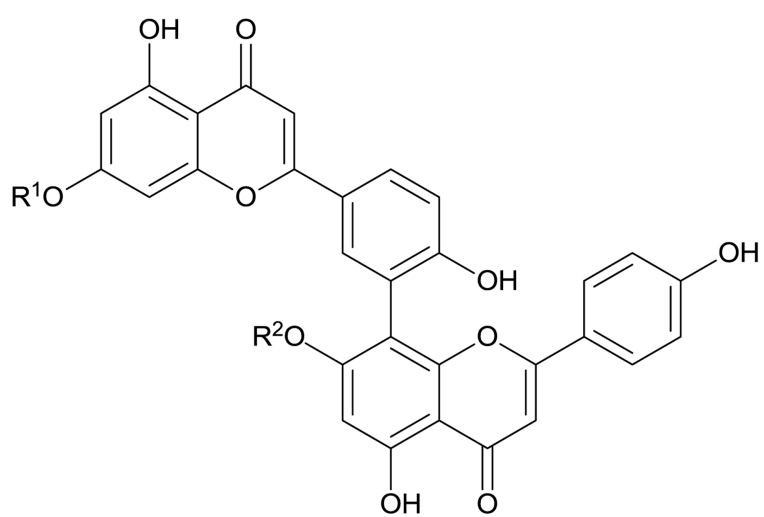
1a: $\mathrm{R}^{1}=\mathrm{H}, \mathrm{R}^{2}=\mathrm{H}$
1f: $\mathrm{R}^{1}=\mathrm{C}_{6} \mathrm{H}_{13}, \mathrm{R}^{2}=\mathrm{C}_{6} \mathrm{H}_{13}$
1b: $R^{1}=\mathrm{H}, \mathrm{R}^{2}=\mathrm{C}_{8} \mathrm{H}_{17}$
1g: $\mathrm{R}^{1}=\mathrm{C}_{8} \mathrm{H}_{17}, \mathrm{R}^{2}=\mathrm{C}_{8} \mathrm{H}_{17}$
1c: $\mathrm{R}^{1}=\mathrm{H}, \mathrm{R}^{2}=\mathrm{C}_{6} \mathrm{H}_{13}$
1h: $\mathrm{R}^{1}=\mathrm{H}, \mathrm{R}^{2}=\mathrm{C}_{10} \mathrm{H}_{21}$
1d: $\mathrm{R}^{1}=\mathrm{H}, \mathrm{R}^{2}=\mathrm{C}_{13} \mathrm{H}_{27}$
1i: $\mathrm{R}^{1}=\mathrm{H}, \mathrm{R}^{2}=\mathrm{C}_{19} \mathrm{H}_{39}$
1e: $R^{1}=H, R^{2}=C_{16} H_{33}$
1j: $R^{1}=H, R^{2}=$ farnesyl

Fig. 1.

Chemical structures of amentoflavone $\mathbf{1 a}$ and analogues $\mathbf{1 b}-\mathbf{j}$. 


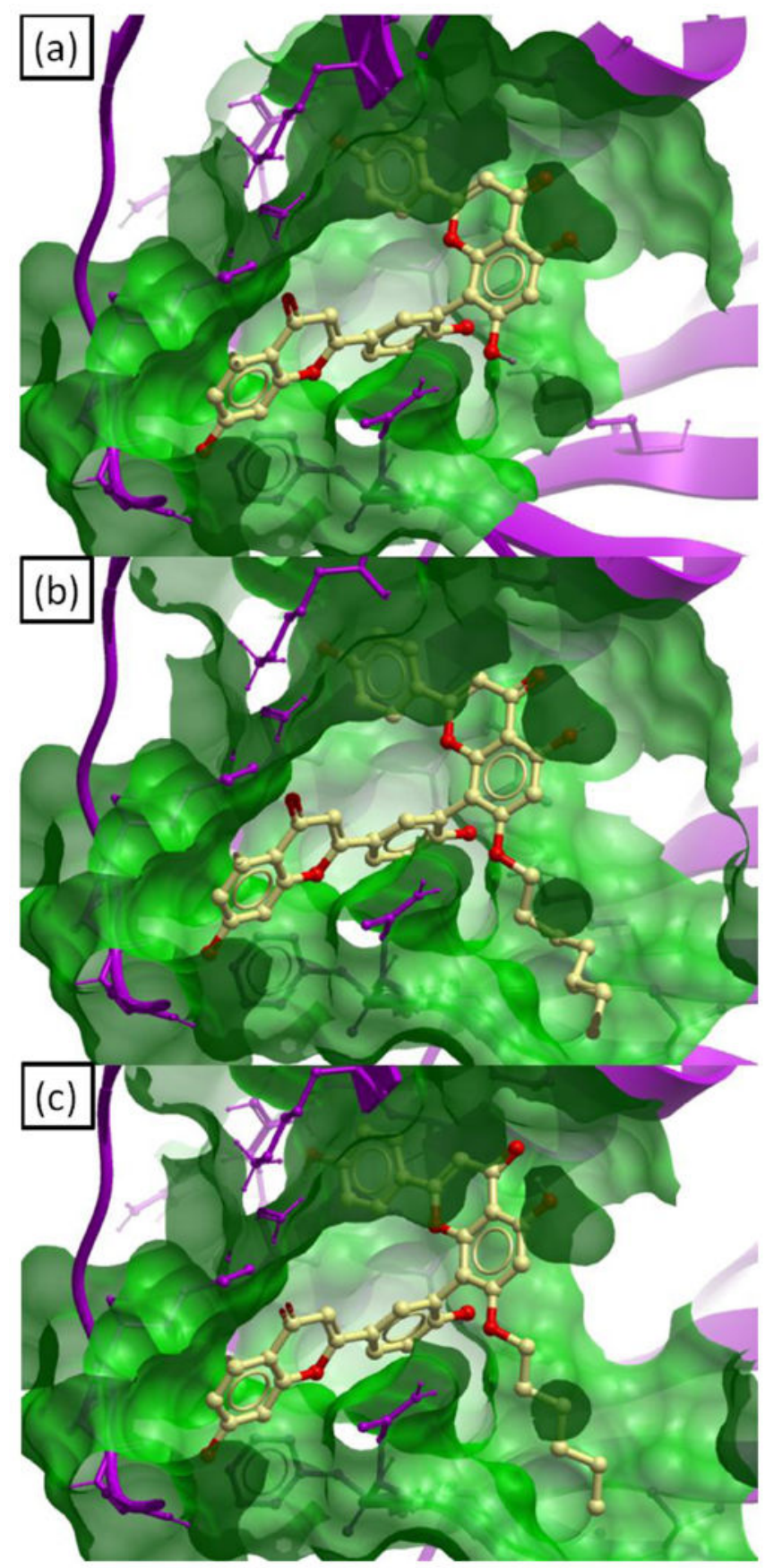

Fig. 2.

Low-energy binding conformations of (a) $1 \mathbf{a}$ and (b) $\mathbf{1 c}$ and (c) $\mathbf{1 b}$ to the DOLPHIN modification of the JAK2 active site as generated by virtual ligand docking. Note the extension of the alkyl chain of $\mathbf{1 b}$ and $\mathbf{1} \mathbf{c}$ into the hydrophobic pocket vacated by the DFG loop. 


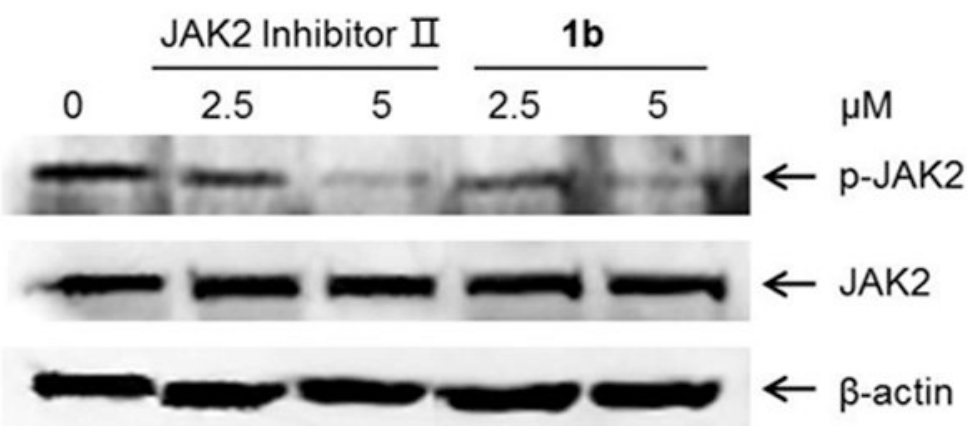

Fig. 3.

Western blot analysis of the effect of compounds $\mathbf{1 b}$ and JAK2 Inhibitor II on JAK2 autophosphorylation in cellulo. HEL cells were incubated with the compounds for $16 \mathrm{~h}$. Protein lysates were electrophoresed and probed for phospho-JAK2 Y1007/Y1008, total JAK2 and total $\beta$-actin levels. Results are representative of three independent experiments. 


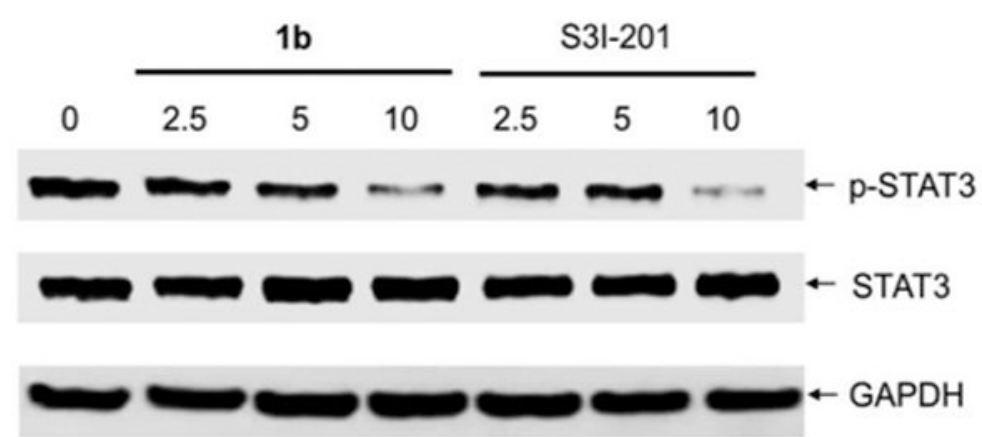

Fig. 4.

Western blot analysis. HEL cells were treated with 1b, positive control compound S3I-201 or vehicle control (0.05\% DMSO) for $24 \mathrm{~h}$. Protein lysates were electrophoresed by SDSPAGE and probed for pSTAT3 (Y705), total STAT3 and GADPH levels. Results are representative of three independent experiments. 

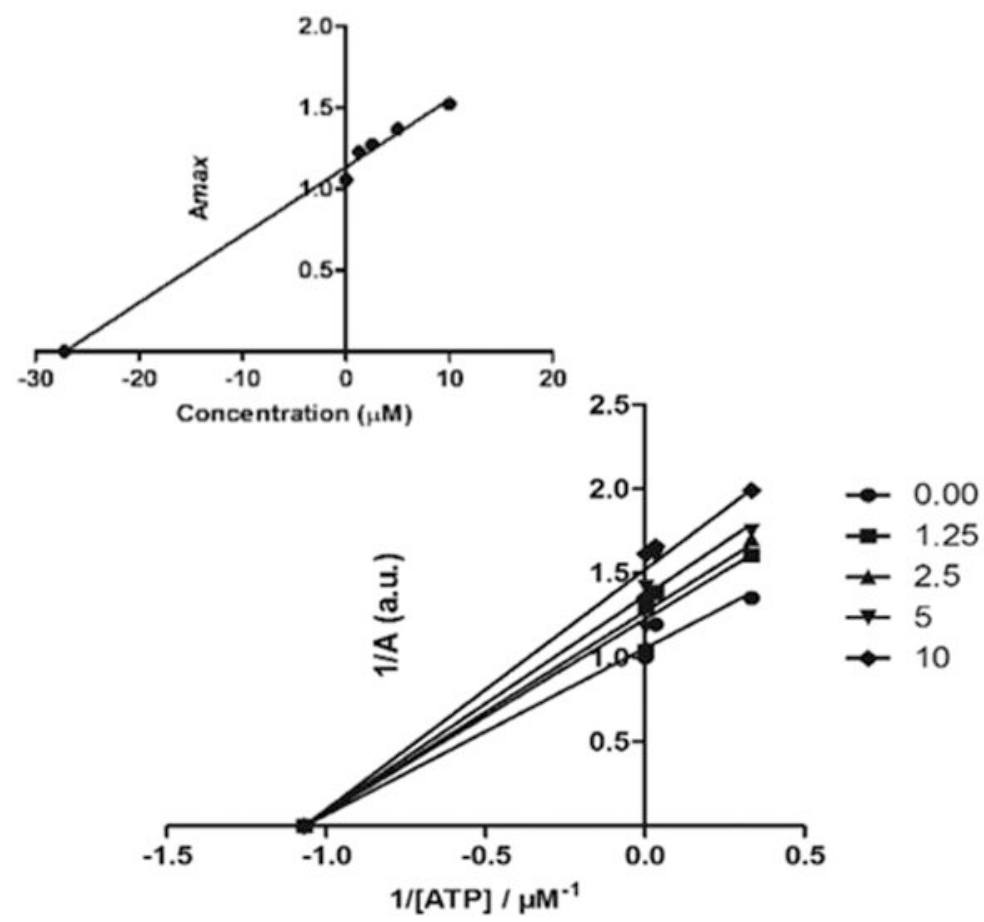

Fig. 5.

Lineweaver-Burke analysis of JAK2 inhibition by $\mathbf{1 b}$ using ELISA. Recombinant JAK2 was incubated with the indicated concentrations of $\mathbf{1 b}$ and biotinylated peptide substrate at room temperature for $30 \mathrm{~min}$. Peptide phosphorylation was detected with anti-phospho-tyrosine primary and horseradish peroxide-conjugated secondary antibody. Inset: Dixon analysis to estimate the $K_{\mathrm{i}}$ value. Results are representative of three independent experiments. 


\section{Table 1}

$\mathrm{EC}_{50}$ values of compounds against viral in $\mathrm{HCV}$-infected cells. Results are representative of three independent experiments.

\begin{tabular}{cc}
\hline Compound & $\mathbf{E C}_{\mathbf{5 0}} / \boldsymbol{\mu M}$ \\
\hline $\mathbf{1 a}$ & $>50$ \\
$\mathbf{1 b}$ & $3.1 \pm 0.8$ \\
$\mathbf{1 c}$ & $>3$ \\
$\mathbf{1 g}$ & $>50$ \\
\hline
\end{tabular}

